\title{
On the trace anomaly of a Weyl fermion in a gauge background
}

\author{
Fiorenzo Bastianelli ${ }^{1,2,3, \mathrm{a}}$, Matteo Broccoli ${ }^{1,3, \mathrm{~b}}$ \\ ${ }^{1}$ Dipartimento di Fisica e Astronomia, Università di Bologna, via Irnerio 46, 40126 Bologna, Italy \\ 2 INFN, Sezione di Bologna, via Irnerio 46, 40126 Bologna, Italy \\ ${ }^{3}$ Max-Planck-Institut für Gravitationsphysik (Albert-Einstein-Institut), Am Mühlenberg 1, Golm, 14476 Potsdam, Germany
}

Received: 30 October 2018 / Accepted: 19 March 2019 / Published online: 1 April 2019

(C) The Author(s) 2019

\begin{abstract}
We study the trace anomaly of a Weyl fermion in an abelian gauge background. Although the presence of the chiral anomaly implies a breakdown of gauge invariance, we find that the trace anomaly can be cast in a gauge invariant form. In particular, we find that it does not contain any odd-parity contribution proportional to the ChernPontryagin density, which would be allowed by the consistency conditions. We perform our calculations using PauliVillars regularization and heat kernel methods. The issue is analogous to the one recently discussed in the literature about the trace anomaly of a Weyl fermion in curved backgrounds.
\end{abstract}

\section{Introduction}

In this paper we study the trace anomaly of a chiral fermion coupled to an abelian gauge field in four dimensions. It is well-known that the model contains an anomaly in the axial gauge symmetry, thus preventing the quantization of the gauge field in a consistent manner. Nevertheless, it is useful to study the explicit structure of the trace anomaly emerging in the axial $U(1)$ background.

One reason to study the problem is that an analogous situation has recently been addressed for a Weyl fermion coupled to gravity. In particular, the presence of an odd-parity term (the Pontryagin density of the curved background) in the trace anomaly has been reported in [1], and further elaborated upon in $[2,3]$. This anomaly was envisaged also in [4], and discussed more recently in [5]. However, there are many indications that such an anomaly cannot be present in the theory of a Weyl fermion. The explicit calculation carried out in [6] confirms this last point of view.

One of the reasons why one does not expect the oddparity contribution to the trace anomaly is that by CPT in four dimensions a left handed fermion has a right handed

\footnotetext{
a e-mail: bastianelli@bo.infn.it

b e-mail: matteo.broccoli2@studio.unibo.it
}

antiparticle, expected to contribute oppositely to any chiral imbalance in the coupling to gravity. To see that, one may cast the quantum field theory of a Weyl fermion as the quantum theory of a Majorana fermion. The latter shows no sign of an odd-parity trace anomaly. Indeed, the functional determinant that arises in a path integral quantization can be regulated using Pauli-Villars Majorana fermions with Majorana mass, so to keep the determinant manifestly real, thereby excluding the appearance of a phase that might produce an anomaly (the odd-parity term would carry an imaginary coefficient) [7]. Recently, this has been verified again using Feynman diagrams [8], which confirms the results of [6]. An additional piece of evidence comes from studies of the 3-point correlation functions of conserved currents in four dimensional CFTs, which exclude odd-parity terms in the correlation function of three stress tensors at non-coinciding points $[9,10]$, seemingly excluding its presence also in the trace anomaly (see however [11]).

Here we analyze the analogous situation of a Weyl fermion coupled to an abelian $U(1)$ gauge background. The theory exhibits a chiral anomaly that implies a breakdown of gauge invariance. It is nevertheless interesting to compute its trace anomaly. Apart from the standard gauge invariant contribution $\left(\sim F^{2}\right)$ and possible gauge noninvariant terms, which as we shall show can be canceled by counterterms, one might expect a contribution from the odd-parity Chern-Pontryagin density $F \tilde{F}$. Indeed the latter satisfies the consistency conditions for trace anomalies. In addition, the fermionic functional determinant is now complex in euclidean space, and thus carries a phase (which is responsible for the known $U(1)$ axial anomaly). On the other hand, the structure of the 3point correlation function of the stress tensor with two $U(1)$ currents in generic CFTs does not allow for odd-parity terms $[9,10]$ that could signal a corresponding anomaly in the trace of the stress tensor in a $U(1)$ background. Apart from a few differences, the case seems analogous to that of the chiral fermion in curved space, and thus it is worth addressing. 
To ascertain the situation we compute explicitly the trace anomaly of a Weyl fermion coupled to a $U(1)$ gauge field. Using a Pauli-Villars regularization we find that no oddparity term emerges in the quantum trace of the stress tensor. We use a Majorana mass for computing the trace anomaly, as this mass term can be covariantized (to curved space) without the need of introducing additional fields of opposite chirality, as required by a Dirac mass. The coupling to gravity (needed only at linear order) is used to treat the metric (or vierbein) as an external source for the stress tensor, and to relate the trace of the latter to a Weyl rescaling of the metric (or vierbein). The manifest covariance of the Majorana mass guarantees that the stress tensor can be kept conserved and symmetric also at the quantum level, i.e. without general coordinate (Einstein) and local Lorentz anomalies. We repeat part of our calculations with a Dirac mass as well. In addition, we calculate also the anomalies of a massless Dirac fermion which, while well-known, serve for comparison and as a test on the scheme adopted. We verify the consistency of the different regularizations, and report the local counterterms that relate them.

We organize the paper as follows. In Sect. 2 we set up the stage and review the lagrangians of the Weyl and Dirac fermions, respectively, and identify the relevant differential operators that enter our regularization schemes. In Sect. 3 we review the method that we choose for computing the chiral and trace anomalies. In Sect. 4 we present our results. We conclude in Sect. 5, confining to the appendices notational conventions, heat kernels formulas, and sample calculations.

\section{Actions and symmetries}

We first present the classical models that we wish to consider, and review their main properties to set up the stage for our calculations. The model of main interest is a massless Weyl fermion coupled to an abelian gauge field. We first describe its symmetries, and then the mass terms to be used in a Pauli-Villars regularization. For comparison, we consider also a massless Dirac fermion coupled to vector and axial abelian gauge fields, a set-up used by Bardeen to compute systematically the anomalies in vector and axial currents [12]. Our notation is commented upon and recapitulated in "Appendix A".

\subsection{The Weyl fermion}

The lagrangian of a left handed Weyl spinor $\lambda$ coupled to a $U$ (1) gauge field is

$\mathscr{L}_{W}=-\bar{\lambda} \gamma^{a}\left(\partial_{a}-i A_{a}\right) \lambda=-\bar{\lambda} \gamma^{a} D_{a}(A) \lambda=-\bar{\lambda} \not D(A) \lambda$ where the chirality of the spinor is defined by the constraint $\gamma^{5} \lambda=\lambda$, or equivalently by $\lambda=\frac{1+\gamma^{5}}{2} \lambda$. It is classically gauge invariant and conformally invariant. Both symmetries become anomalous at the quantum level.

In the following we find it convenient to use the charge conjugated spinor $\lambda_{c}$, which has the opposite chirality of $\lambda$

$\lambda_{c}=C^{-1} \bar{\lambda}^{T}, \quad \gamma^{5} \lambda_{c}=-\lambda_{c}$.

The lagrangian can be cast in equivalent forms using $\lambda_{c}$ rather then $\bar{\lambda}$

$$
\begin{aligned}
\mathscr{L}_{W} & =\lambda_{c}^{T} C \not D(A) \lambda=\lambda^{T} C \not D(-A) \lambda_{c} \\
& =\frac{1}{2}\left(\lambda_{c}^{T} C \not D(A) \lambda+\lambda^{T} C \not D(-A) \lambda_{c}\right)
\end{aligned}
$$

where the last two forms are valid up to boundary terms (we perform partial integrations in the action and drop boundary terms). We use the last form in our calculations.

The gauge transformations can be written as

$$
\left\{\begin{aligned}
\lambda(x) & \rightarrow \lambda^{\prime}(x)=e^{i \alpha(x)} \lambda(x) \\
\bar{\lambda}(x) & \rightarrow \bar{\lambda}^{\prime}(x)=e^{-i \alpha(x)} \bar{\lambda}(x) \\
\lambda_{c}(x) & \rightarrow \lambda_{c}^{\prime}(x)=e^{-i \alpha(x)} \lambda_{c}(x) \\
A_{a}(x) & \rightarrow A_{a}^{\prime}(x)=A_{a}(x)+\partial_{a} \alpha(x)
\end{aligned}\right.
$$

and the action $S_{W}=\int d^{4} x \mathscr{L}_{W}$ is gauge invariant. Recall also that $A_{a}$ can be used as an external source for the current

$$
J^{a}=i \bar{\lambda} \gamma^{a} \lambda
$$

Varying in the action only $A_{a}$ with a gauge transformation with infinitesimal parameter $\alpha(x)$ produces

$\delta_{\alpha}^{(A)} S_{W}=-\int d^{4} x \alpha(x) \partial_{a} J^{a}(x)$

and the full gauge symmetry $\left(\delta_{\alpha} S_{W}=0\right)$ guarantees that the $U(1)$ current is conserved on-shell (i.e. using the fermion equations of motion)

$\partial_{a} J^{a}(x)=0$.

Similarly, one may check that the action is classically conformal invariant and that the stress tensor has a vanishing trace. To see this one couples the model to gravity by introducing the vierbein $e_{\mu}{ }^{a}$ (and related spin connection $\omega_{\mu}{ }^{a b}$ ), and realizes that the action is invariant under general coordinate, local Lorentz, and Weyl transformations. The stress tensor, or energy momentum tensor, is defined as usual by

$T^{\mu a}(x)=\frac{1}{e} \frac{\delta S_{W}}{\delta e_{\mu a}(x)}$

where $e$ is the determinant of the vierbein, and is covariantly conserved, symmetric, and traceless on-shell, as con- 
sequence of diffeomorphisms, local Lorentz invariance, and Weyl symmetry, respectively

$\nabla_{\mu} T^{\mu a}=0, \quad T_{a b}=T_{b a}, \quad T^{a}{ }_{a}=0$

(indices are made "curved" or "flat" by using the vierbein and its inverse). The vierbein can be used as an external source for the stress tensor, and an infinitesimal Weyl transformation on the vierbein acts as a source for the trace $T^{a}{ }_{a}$. In the following we only need a linearized coupling to gravity to produce a single insertion of the stress tensor in correlation functions. Otherwise, we are interested in flat space results only. In any case, the full coupling to gravity reads

$\mathscr{L}_{W}=-e \bar{\lambda} \gamma^{\mu} \nabla_{\mu} \lambda$

where $\gamma^{\mu}=e^{\mu}{ }_{a} \gamma^{a}$ are the gamma matrices with curved indices, $e^{\mu}{ }_{a}$ is the inverse vierbein, and $\nabla_{\mu}$ is the covariant derivative containing both the $U(1)$ gauge field $A_{\mu}$ and spin connection $\omega_{\mu a b}$

$\nabla_{\mu}=\partial_{\mu}-i A_{\mu}+\frac{1}{4} \omega_{\mu a b} \gamma^{a} \gamma^{b}$.

The local Weyl symmetry is given by

$\left\{\begin{aligned} \lambda(x) & \rightarrow \lambda^{\prime}(x)=e^{-\frac{3}{2} \sigma(x)} \lambda(x) \\ \bar{\lambda}(x) & \rightarrow \bar{\lambda}^{\prime}(x)=e^{-\frac{3}{2} \sigma(x)} \bar{\lambda}(x) \\ A_{a}(x) & \rightarrow A_{a}^{\prime}(x)=A_{a}(x) \\ e_{\mu}{ }^{a}(x) & \rightarrow e^{\prime}{ }_{\mu}{ }^{a}(x)=e^{\sigma(x)} e_{\mu}{ }^{a}(x)\end{aligned}\right.$

where $\sigma(x)$ is an arbitrary function. Varying in the action only the vierbein with an infinitesimal Weyl transformation produces the trace of the stress tensor

$\delta_{\sigma}^{(e)} S_{W}=-\int d^{4} x e \sigma(x) T^{a}{ }_{a}(x)$

and the full Weyl symmetry of the action $\left(\delta_{\sigma} S_{W}=0\right)$ guarantees that it is traceless on-shell

$T^{a}{ }_{a}(x)=0$.

For completeness, we record the form of the stress tensor in flat space emerging from the previous considerations, simplified by using the equations of motion,

$T_{a b}=\frac{1}{4} \bar{\lambda}\left(\gamma_{a} \stackrel{\leftrightarrow}{D}_{b}+\gamma_{b} \stackrel{\leftrightarrow}{D}_{a}\right) \lambda$

where $\stackrel{\leftrightarrow}{D}_{a}=D_{a}-\overleftarrow{D}_{a}$ (in terms of the gauge covariant derivative). It is traceless on-shell.

\subsubsection{Mass terms}

To compute the anomalies in the quantum theory we regularize the latter introducing massive Pauli-Villars (PV) fields, with the anomalies eventually coming from the noninvariance of the mass term. For the massless Weyl fermion, one can take as PV field a Weyl fermion of the same chirality, with a Majorana mass added. The mass term is Lorentz invariant, but breaks the gauge and conformal symmetries. It takes many equivalent forms

$$
\begin{aligned}
\Delta_{M} \mathscr{L}_{W} & =\frac{M}{2}\left(\lambda^{T} C \lambda+\text { h.c. }\right) \\
& =\frac{M}{2}\left(\lambda^{T} C \lambda-\bar{\lambda} C^{-1} \bar{\lambda}^{T}\right) \\
& =\frac{M}{2}\left(\lambda^{T} C \lambda+\lambda_{c}^{T} C \lambda_{c}\right)
\end{aligned}
$$

where h.c. denotes the hermitian conjugate and $M$ is a real mass parameter. Since the charge conjugation matrix $C$ is antisymmetric this term is nonvanishing for an anticommuting spinor. ${ }^{1}$

Casting the full massive PV action $\mathscr{L}_{P V}=\mathscr{L}_{W}+\Delta_{M} \mathscr{L}_{W}$ in the compact form

$\mathscr{L}_{P V}=\frac{1}{2} \phi^{T} T \mathscr{O} \phi+\frac{1}{2} M \phi^{T} T \phi$,

where $\phi$ is a column vector containing both $\lambda$ and $\lambda_{c}$ (thus $\phi$ is a 8 dimensional vector)

$\phi=\left(\begin{array}{c}\lambda \\ \lambda_{c}\end{array}\right)$,

permits the identification of the operators

$T \mathscr{O}=\left(\begin{array}{cc}0 & C D(-A) P_{R} \\ C \not D(A) P_{L} & 0\end{array}\right), \quad T=\left(\begin{array}{cc}C P_{L} & 0 \\ 0 & C P_{R}\end{array}\right)$

and

$$
\begin{aligned}
\mathscr{O} & =\left(\begin{array}{cc}
0 & \not D(-A) P_{R} \\
D(A) P_{L} & 0
\end{array}\right), \\
\mathscr{O}^{2} & =\left(\begin{array}{cc}
D(-A) \not D(A) P_{L} & 0 \\
0 & \not D(A) \not D(-A) P_{R}
\end{array}\right) .
\end{aligned}
$$

The latter will be used in our anomaly calculations. The chiral projectors $P_{L}$ and $P_{R}$

$P_{L}=\frac{\mathbb{1}+\gamma^{5}}{2}, \quad P_{R}=\frac{\mathbb{1}-\gamma^{5}}{2}$

have been introduced to stress that the matrix $T$ is not invertible in the full 8 dimensional space on which $\phi$ lives. An

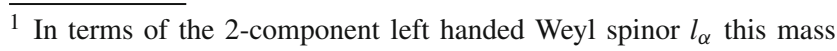
term reads as

$\Delta_{M} \mathscr{L}_{W}=\frac{M}{2}\left(l_{\alpha}\left(-i \sigma^{2}\right)^{\alpha \beta} l_{\beta}+l_{\dot{\alpha}}^{*}\left(i \sigma^{2}\right)^{\dot{\alpha} \dot{\beta}} l_{\dot{\beta}}^{*}\right)$

and it does not contain any other spinor apart from $l_{\alpha}$ and its complex conjugate $l_{\dot{\alpha}}^{*}$. In the chiral representation of the gamma matrices the 2-component spinor $l_{\alpha}$ sits inside $\lambda$, as in Eq. (A.12). 
advantage of the Majorana mass term is that it can be constructed without the need of introducing extra degrees of freedom (as required by the Dirac mass term discussed below). Moreover, it can be covariantized under Einstein (general coordinate) and local Lorentz symmetries. The covariantization is achieved by multiplying it with the determinant of the vierbein $e$

$\Delta_{M} \mathscr{L}_{W}=\frac{e M}{2}\left(\lambda^{T} C \lambda+\lambda_{c}^{T} C \lambda_{c}\right)$.

An alternative mass term is the Dirac mass. To use it one must introduce in addition also an uncoupled right handed PV fermion $\rho$ (satisfying $\rho=P_{R} \rho$ ), so that the full massive PV lagrangian reads

$$
\tilde{\mathscr{L}}_{P V}=-\bar{\lambda} \not D(A) \lambda-\bar{\rho} \not \rho-M(\bar{\lambda} \rho+\bar{\rho} \lambda)
$$

or, equivalently,

$$
\begin{aligned}
\tilde{\mathscr{L}}_{P V}= & \frac{1}{2}\left(\lambda_{c}^{T} C \not D(A) \lambda+\lambda^{T} C \not D(-A) \lambda_{c}\right) \\
& +\frac{1}{2}\left(\rho_{c}^{T} C \not \rho \rho+\rho^{T} C \not \partial \rho_{c}\right) \\
& +\frac{M}{2}\left(\lambda_{c}^{T} C \rho+\rho^{T} C \lambda_{c}+\rho_{c}^{T} C \lambda+\lambda^{T} C \rho_{c}\right) .
\end{aligned}
$$

The latter expression allows to cast the PV lagrangian in the general form (18) with

$\phi=\left(\begin{array}{c}\lambda \\ \lambda_{c} \\ \rho \\ \rho_{c}\end{array}\right)$

where each entry is a 4 dimensional Dirac spinor (with chiral projectors attached), and one finds

$$
\begin{aligned}
& T \mathscr{O}=\left(\begin{array}{cccc}
0 & C \not D(-A) P_{R} & 0 & 0 \\
C \not D(A) P_{L} & 0 & 0 & 0 \\
0 & 0 & 0 & C \not \partial P_{L} \\
0 & 0 & C \not \partial P_{R} & 0
\end{array}\right) \\
& T=\left(\begin{array}{cccc}
0 & 0 & 0 & C P_{L} \\
0 & 0 & C P_{R} & 0 \\
0 & C P_{R} & 0 & 0 \\
C P_{L} & 0 & 0 & 0
\end{array}\right) \\
& \mathscr{O}=\left(\begin{array}{cccc}
0 & 0 & \not \partial P_{R} & 0 \\
0 & 0 & 0 & \not \partial P_{L} \\
D(A) P_{L} & 0 & 0 & 0 \\
0 & \not D(-A) P_{R} & 0 & 0
\end{array}\right) \\
& \mathscr{O}^{2}=\left(\begin{array}{cccc}
\not \supset D(A) P_{L} & 0 & 0 & 0 \\
0 & \not \partial D(-A) P_{R} & 0 & 0 \\
0 & 0 & \not D(A) \not \partial P_{R} & 0 \\
0 & 0 & 0 & \not D(-A) \not \partial P_{L}
\end{array}\right) \text {. }
\end{aligned}
$$

The differential operators in $\mathscr{O}^{2}$ have appeared also in [13], where definitions for the determinant of a chiral Dirac oper- ator were studied with the purpose of addressing the chiral anomalies.

A drawback of the Dirac mass term, as regulator of the Weyl theory, is that one cannot covariantize it while keeping the auxiliary right handed spinor $\rho$ free in the kinetic term (it cannot be coupled to gravity, otherwise it would not regulate properly the original chiral theory). One can still use the regularization keeping $\rho$ free in the kinetic term but, as the mass term breaks the Einstein and local Lorentz symmetries explicitly, one would get anomalies in the conservation $\left(\partial_{a} T^{a b}\right)$ and antisymmetric part $\left(T^{[a b]}\right)$ of the stress tensor. Then, one is forced to study the counterterms to reinstate conservation and symmetry of the stress tensor (it can always be done in 4 dimensions $[7,14]$ ), and eventually check which trace anomaly one is left with. As this is rather laborious, we do not use this mass term to calculate the trace anomaly in the Weyl theory. ${ }^{2}$

\subsection{The Dirac fermion}

We consider also the more general model of a massless Dirac fermion coupled to vector and axial $U(1)$ gauge fields $A_{a}$ and $B_{a}$. The lagrangian is

$$
\begin{aligned}
\mathscr{L}_{D} & =-\bar{\psi} \gamma^{a}\left(\partial_{a}-i A_{a}-i B_{a} \gamma^{5}\right) \psi=-\bar{\psi} \not D(A, B) \psi \\
& =\frac{1}{2} \psi_{c}^{T} C \not D(A, B) \psi+\frac{1}{2} \psi^{T} C \not D(-A, B) \psi_{c}
\end{aligned}
$$

where the last form is valid up to boundary terms. A chiral projector emerges when $A_{a}= \pm B_{a}$, and we use this model to address again the issue of the chiral fermion in flat space (the limit $A_{a}=B_{a} \rightarrow \frac{A_{a}}{2}$ reproduces the massless part of (24)).

The lagrangian is invariant under the local $U(1)_{V}$ vector transformations

$$
\left\{\begin{array}{l}
\psi(x) \rightarrow \psi^{\prime}(x)=e^{i \alpha(x)} \psi(x) \\
\bar{\psi}(x) \rightarrow \bar{\psi}^{\prime}(x)=e^{-i \alpha(x)} \bar{\psi}(x) \\
\psi_{c}(x) \rightarrow \psi_{c}^{\prime}(x)=e^{-i \alpha(x)} \psi_{c}(x) \\
A_{a}(x) \rightarrow A_{a}^{\prime}(x)=A_{a}(x)+\partial_{a} \alpha(x) \\
B_{a}(x) \rightarrow B_{a}^{\prime}(x)=B_{a}(x)
\end{array}\right.
$$

and local $U(1)_{A}$ axial transformations

$$
\left\{\begin{aligned}
\psi(x) & \rightarrow \psi^{\prime}(x)=e^{i \beta(x) \gamma^{5}} \psi(x) \\
\bar{\psi}(x) & \rightarrow \bar{\psi}^{\prime}(x)=\bar{\psi}(x) e^{i \beta(x) \gamma^{5}} \\
\psi_{c}(x) & \rightarrow \psi_{c}^{\prime}(x)=e^{i \beta(x) \gamma^{5}} \psi_{c}(x) \\
A_{a}(x) & \rightarrow A_{a}^{\prime}(x)=A_{a}(x) \\
B_{a}(x) & \rightarrow B_{a}^{\prime}(x)=B_{a}(x)+\partial_{a} \beta(x) .
\end{aligned}\right.
$$

2 A possibility to simplify the calculation would be to use the axial metric background introduced in $[2,3]$, but we will not follow this direction either. 
Again one can use $A_{a}$ and $B_{a}$ as sources for $J^{a}=i \bar{\psi} \gamma^{a} \psi$ and $J_{5}^{a}=i \bar{\psi} \gamma^{a} \gamma^{5} \psi$, respectively. Under infinitesimal variation of these external sources one finds

$\delta_{\alpha}^{(A)} S_{D}=-\int d^{4} x \alpha(x) \partial_{a} J^{a}(x)$

$\delta_{\beta}^{(B)} S_{D}=-\int d^{4} x \beta(x) \partial_{a} J_{5}^{a}(x)$

and the classical gauge symmetries imply that $J^{a}$ and $J_{5}^{a}$ are conserved on-shell

$\partial_{a} J^{a}(x)=0$

$\partial_{a} J_{5}^{a}(x)=0$.

A coupling to gravity shows that the stress tensor is traceless because of the Weyl symmetry. The Weyl transformations rules have the same form as in (12), with $B_{a}$ left invariant. An infinitesimal Weyl variation on the vierbein produces the trace of the stress tensor

$\delta_{\sigma}^{(e)} S_{D}=-\int d^{4} x e \sigma(x) T_{a}^{a}(x)$.

and the Weyl symmetry implies that it vanishes on-shell

$T^{a}{ }_{a}(x)=0$.

\subsubsection{Mass terms}

To regulate the one-loop graphs we introduces massive PV fields. The standard Dirac mass term

$\Delta_{M} \mathscr{L}_{D}=-M \bar{\psi} \psi=\frac{M}{2}\left(\psi_{c}^{T} C \psi+\psi^{T} C \psi_{c}\right)$

preserves vector gauge invariance, and casting the PV lagrangian $\mathscr{L}_{P V}=\mathscr{L}_{D}+\Delta_{M} \mathscr{L}_{D}$ in the form (18), now with $\phi=\left(\begin{array}{c}\psi \\ \psi_{c}\end{array}\right)$, allows to recognize the operators

$T \mathscr{O}=\left(\begin{array}{cc}0 & C \not D(-A, B) \\ C \not D(A, B) & 0\end{array}\right), \quad T=\left(\begin{array}{ll}0 & C \\ C & 0\end{array}\right)$

and

$$
\begin{aligned}
\mathscr{O} & =\left(\begin{array}{cc}
D(A, B) & 0 \\
0 & \not D(-A, B)
\end{array}\right), \\
\mathscr{O}^{2} & =\left(\begin{array}{cc}
D(A, B)^{2} & 0 \\
0 & \not D(-A, B)^{2}
\end{array}\right) .
\end{aligned}
$$

This mass term mixes the two chiral parts $\lambda$ and $\rho$ of the Dirac fermion $\psi=\lambda+\rho$, see Eqs. (24) or (25) that makes it visible. After covariantization to gravity the decoupling of the two chiralities is not easily achievable, and relations between the trace anomaly of a Dirac fermion and the trace anomaly of a Weyl fermion cannot be studied directly by using a Dirac mass in the PV regularization.
Thus, it is useful to consider a Majorana mass as well. It breaks both vector and axial symmetries

$\tilde{\Delta}_{M} \mathscr{L}_{D}=\frac{M}{2}\left(\psi^{T} C \psi+\right.$ h.c. $)=\frac{M}{2}\left(\psi^{T} C \psi+\psi_{c}^{T} C \psi_{c}\right)$

and one finds from the alternative PV lagrangian $\tilde{\mathscr{L}}_{P V}=$ $\mathscr{L}_{D}+\tilde{\Delta}_{M} \mathscr{L}_{D}$ the operators

$T \mathscr{O}=\left(\begin{array}{cc}0 & C \not D(-A, B) \\ C \not D(A, B) & 0\end{array}\right), \quad T=\left(\begin{array}{ll}C & 0 \\ 0 & C\end{array}\right)$

and

$$
\begin{aligned}
\mathscr{O} & =\left(\begin{array}{cc}
0 & \not D(-A, B) \\
D(A, B) & 0
\end{array}\right), \\
\mathscr{O}^{2} & =\left(\begin{array}{cc}
D(-A, B) \not D(A, B) & 0 \\
0 & D(A, B) \not D(-A, B)
\end{array}\right) .
\end{aligned}
$$

Covariantization to gravity does not mix the chiral parts of the Dirac fermion, and a decoupling limit to the chiral theory of a Weyl fermion $\lambda$ is now attainable.

\section{Regulators and consistent anomalies}

To compute the anomalies we employ a Pauli-Villars regularization [15]. Following the scheme of Refs. [16,17] we cast the calculation in the same form as the one obtained by Fujikawa in analyzing the measure of the path integral $[18,19]$. This makes it easier to use heat kernel formulas $[20,21]$ to evaluate the anomalies. At the same time, the method guarantees one to obtain consistent anomalies, i.e. anomalies that satisfy the consistency conditions $[22,23]$.

Let us review the scheme of Ref. [16]. One considers a lagrangian for a field $\varphi$

$\mathscr{L}=\frac{1}{2} \varphi^{T} T \mathscr{O} \varphi$

which is invariant under a linear symmetry

$\delta \varphi=K \varphi$

that generically acts also on the operator $T \mathscr{O}$, as it may depend on background fields. The one-loop effective action can be regulated by subtracting a loop of a massive PV field $\phi$ with action

$\mathscr{L}_{P V}=\frac{1}{2} \phi^{T} T \mathscr{O} \phi+\frac{1}{2} M \phi^{T} T \phi$ 
where $M$ is a real parameter. ${ }^{3}$ The mass term identifies the operator $T$, that in turn allows to find the operator $\mathscr{O}$. As we shall see, in fermionic theories with a first order differential operator $\mathscr{O}$ in the kinetic term, the operator $\mathscr{O}^{2}$ acts as a regulator in the final formula for the anomaly. The invariance of the original action extends to an invariance of the massless part of the PV action by defining

$\delta \phi=K \phi$

so that only the mass term may break the symmetry

$$
\begin{aligned}
\delta \mathscr{L}_{P V} & =\frac{1}{2} M \phi^{T}\left(T K+K^{T} T+\delta T\right) \phi \\
& =M \phi^{T}\left(T K+\frac{1}{2} \delta T\right) \phi .
\end{aligned}
$$

The path integral $Z$ and the one-loop effective action $\Gamma$ are regulated by the PV field

$$
\begin{aligned}
Z & =e^{i \Gamma}=\int D \varphi e^{i S} \Rightarrow Z=e^{i \Gamma} \\
& =\int D \varphi D \phi e^{i\left(S+S_{P V}\right)}
\end{aligned}
$$

where it is understood that one should take the $M \rightarrow \infty$ limit, with all divergences canceled as explained in the footnote. The anomalous response of the path integral under a symmetry is due to the PV mass term only, as one can define the measure of the PV field so to make the whole path integral measure invariant [16]. In a hypercondensed notation, where a term like $\phi^{T} \phi$ includes in the sum of the indices a spacetime integration as well, a lagrangian like the one in (46) is equivalent to the action, and one may compute the symmetry variation of the regulated path integral to obtain

$$
\begin{aligned}
i \delta \Gamma & =i\langle\delta S\rangle=\lim _{M \rightarrow \infty} i M\left\langle\phi^{T}\left(T K+\frac{1}{2} \delta T\right) \phi\right\rangle \\
& =-\lim _{M \rightarrow \infty} \operatorname{Tr}\left[\left(K+\frac{1}{2} T^{-1} \delta T\right)\left(1+\frac{\mathscr{O}}{M}\right)^{-1}\right]
\end{aligned}
$$

where brackets $\langle\ldots\rangle$ denote normalized correlation functions. For our purposes, it is convenient to cast it in an equivalent form [17]

$$
\begin{aligned}
i \delta \Gamma & =i\langle\delta S\rangle \\
& =-\lim _{M \rightarrow \infty} \operatorname{Tr}\left[\left(K+\frac{1}{2} T^{-1} \delta T+\frac{1}{2} \frac{\delta \mathscr{O}}{M}\right)\left(1-\frac{\mathscr{O}^{2}}{M^{2}}\right)^{-1}\right]
\end{aligned}
$$

\footnotetext{
${ }^{3}$ To be precise, one should employ a set of PV fields with mass $M_{i}$ and relative weight $c_{i}$ in the loop to be able to regulate and cancel all possible one-loop divergences [15]. For simplicity, we consider only one PV field with relative weight $c=-1$, as this is enough for our purposes. The weight $c=-1$ means that we are subtracting a massive $\mathrm{PV}$ loop from the original one.
}

obtained by inserting the identity $1=\left(1-\frac{\mathscr{O}}{M}\right)\left(1-\frac{\mathscr{O}}{M}\right)^{-1}$ and using the invariance of the massless action

$\delta \mathscr{L}=\varphi^{T}\left(T \mathscr{O} K+\frac{1}{2} \delta T \mathscr{O}+\frac{1}{2} T \delta \mathscr{O}\right) \varphi=0$.

In deriving these expressions, we have considered a fermionic theory, used the PV propagator

$\left\langle\phi \phi^{T}\right\rangle=\frac{i}{T \mathscr{O}+T M}$,

taken into account the opposite sign for the PV field in the loop, and considered an invertible mass matrix $T$.

In the limit $M \rightarrow \infty$ the regulating term $\left(1-\frac{\mathscr{O}^{2}}{M^{2}}\right)^{-1}$ inside (51) can be replaced by $e^{\frac{\sigma^{2}}{M^{2}}}$. This is allowed as, for the purpose of extracting the limit, these regulators cut off the ultraviolet frequencies in an equivalent way (we assume that $\mathscr{O}^{2}$ is negative definite after a Wick rotation to euclidean space) [16]. Clearly, if one finds a symmetrical mass term, then the symmetry would remain automatically anomaly free.

Heat kernel formulas may now be directly applied. Denoting

$J=K+\frac{1}{2} T^{-1} \delta T+\frac{1}{2} \frac{\delta \mathscr{O}}{M}, \quad R=-\mathscr{O}^{2}$

the anomaly is related to the trace of the heat kernel of the regulator $R$ with an insertion of $J$

$i \delta \Gamma=i\langle\delta S\rangle=-\lim _{M \rightarrow \infty} \operatorname{Tr}\left[J e^{-\frac{R}{M^{2}}}\right]$.

This has the same form that appears in Fujikawa's method for computing anomalies $[18,19]$, where $J$ is the infinitesimal part of the fermionic jacobian arising from a change of the path integral variables under a symmetry transformation, and $R$ is the regulator. The limit extracts the mass independent term (negative powers of the mass vanish in the limit, while positive (diverging) powers are made to cancel by using additional PV fields). The PV method guarantees that the regulator $R$ together with $J$ produces consistent anomalies, which follows from the fact that one is computing directly the variation of the effective action.

The heat kernel formulas that we need in the anomaly calculation are well-known, and we report them in "Appendix B" using a minkowskian time. In particular, in four dimensions we need the Seeley-DeWitt coefficients $a_{2}(R)$, corresponding to the various regulators $R$ associated to the fields assembled into $\phi$. These are the only coefficients that survive in the limit $M \rightarrow \infty$ (as said, diverging pieces are removed by the PV renomalization). Running through the various cases presented in the previous section, we extract the "jacobians" $J$ and regulators $R$ to find the structure of the anomalies. For the Weyl theory we find 


$$
\begin{aligned}
& \partial_{a}\left\langle J^{a}\right\rangle=\frac{i}{(4 \pi)^{2}}\left[\operatorname{tr}\left[P_{L} a_{2}\left(R_{\lambda}\right)\right]-\operatorname{tr}\left[P_{R} a_{2}\left(R_{\lambda_{c}}\right)\right]\right] \\
& \left\langle T^{a}{ }_{a}\right\rangle=-\frac{1}{2(4 \pi)^{2}}\left[\operatorname{tr}\left[P_{L} a_{2}\left(R_{\lambda}\right)\right]+\operatorname{tr}\left[P_{R} a_{2}\left(R_{\lambda_{c}}\right)\right]\right] .
\end{aligned}
$$

These formulas are obtained by considering that for the $U(1)$ symmetry the jacobian $J$ in (54) is found from the symmetry transformations of $\lambda$ and $\lambda_{c}$ in (4)

$J=\left(\begin{array}{cc}i \alpha P_{L} & 0 \\ 0 & -i \alpha P_{R}\end{array}\right)$.

Only $K$ contributes, as $\delta T$ vanishes as well as the contribution from $\delta \mathscr{O}$ (it vanishes after taking the traces in (56), as will be checked in the next section). The infinitesimal parameter $\alpha$ is eventually factorized away from (55) to obtain the local form in (56). In computing $J$ from (54), it is enough to check that the mass matrix $T$ is invertible on the relevant chiral spaces (extracted by the projectors $P_{L}$ and $P_{R}$ ). For the Weyl symmetry one uses instead the transformation laws in (12) to find

$J=\left(\begin{array}{cc}\frac{1}{2} \sigma P_{L} & 0 \\ 0 & \frac{1}{2} \sigma P_{R}\end{array}\right)$,

where it is now crucial to consider that the covariant (under gravity) extension of the mass terms contains a factor of $e$, see Eq. (23), which brings in a contribution from $\frac{1}{2} T^{-1} \delta T$ to $J$. This contribution is necessary to guarantee that general coordinate invariance is kept anomaly free in the regularization $(\delta \mathscr{O}$ is neglected again for the same reason as before). The infinitesimal Weyl parameter $\sigma$ is then factorized away from (55) to obtain the second equation in (56).

Proceeding in a similar way, we find for the Dirac model

$$
\begin{aligned}
& \partial_{a}\left\langle J^{a}\right\rangle=\frac{i}{(4 \pi)^{2}}\left[\operatorname{tr} a_{2}\left(R_{\psi}\right)-\operatorname{tr} a_{2}\left(R_{\psi_{c}}\right)\right] \\
& \partial_{a}\left\langle J_{5}^{a}\right\rangle=\frac{i}{(4 \pi)^{2}}\left[\operatorname{tr}\left[\gamma^{5} a_{2}\left(R_{\psi}\right)\right]+\operatorname{tr} \gamma^{5}\left[a_{2}\left(R_{\psi_{c}}\right)\right]\right] \\
& \left\langle T^{a}{ }_{a}\right\rangle=-\frac{1}{2(4 \pi)^{2}}\left[\operatorname{tr} a_{2}\left(R_{\psi}\right)+\operatorname{tr} a_{2}\left(R_{\psi_{c}}\right)\right] .
\end{aligned}
$$

All remaining traces are traces on the gamma matrices taken in the standard four dimensional Dirac spinor space.

\section{Anomalies}

In this section we compute systematically the chiral and trace anomalies for the Weyl and Dirac theories described earlier. We use, when applicable, two different versions of the Pauli-Villars regularization with different mass terms. We verify that the final results are consistent with each other, and coincide after taking into account the variation of local counterterms.

\subsection{Chiral and trace anomalies of a Weyl fermion}

We consider first the case of a Weyl fermion.

\subsubsection{PV regularization with Majorana mass}

The regularization of the Weyl fermion coupled to an abelian gauge field is achieved in the most minimal way by using a PV fermion of the same chirality with the Majorana mass term in Eq. (16) added. This set-up was already used in [6] to address the case of a Weyl fermion in a gravitational background, but without the abelian gauge coupling. The mass term is Lorentz invariant and does not introduce additional chiralities, but breaks the gauge and conformal (Weyl) symmetries. Therefore, one expects chiral and trace anomalies.

To obtain the anomalies we have to compute the expressions in (56) with the regulators contained inside $\mathscr{O}^{2}$ of Eq. (21). They read

$$
\begin{aligned}
R_{\lambda} & =-\not D(-A) \not D(A) P_{L} \\
R_{\lambda_{c}} & =-\not D(A) \not D(-A) P_{R} .
\end{aligned}
$$

Using the Seeley-DeWitt coefficients $a_{2}$ of these regulators we find the chiral anomaly

$\partial_{a}\left\langle J^{a}\right\rangle=\frac{1}{(4 \pi)^{2}}\left(\frac{1}{6} \varepsilon^{a b c d} F_{a b} F_{c d}-\frac{8}{3} \partial_{a}\left(A^{a} A^{2}\right)+\frac{2}{3} \square(\partial A)\right)$

where $F_{a b}=\partial_{a} A_{b}-\partial_{b} A_{a}$ (see "Appendix C" for an outline of the calculation). It contains normal-parity terms that can be canceled by the gauge variation of the local counterterm

$\Gamma_{1}=\int \frac{d^{4} x}{(4 \pi)^{2}}\left(\frac{2}{3} A^{4}-\frac{1}{3} A^{a} \square A_{a}\right)$,

so that the chiral gauge anomaly takes the form

$\partial_{a}\left\langle J^{a}\right\rangle=\frac{1}{96 \pi^{2}} \varepsilon^{a b c d} F_{a b} F_{c d}$.

This is the standard result.

Similarly, we compute the trace anomaly, given by

$$
\left\langle T_{a}^{a}{ }_{a}\right\rangle=-\frac{1}{(4 \pi)^{2}}\left(\frac{2}{3}\left(\partial_{a} A_{b}\right)\left(\partial^{a} A^{b}\right)-\frac{2}{3}(\partial A)^{2}-\frac{2}{3} \square A^{2}\right) .
$$

It does not contain any odd-parity contribution. Gauge invariance is broken by the chiral anomaly, but we find that the trace anomaly can be cast in a gauge invariant form by varying a local counterterm with a Weyl transformation (and then restricting to flat space). The (gravity covariant but gauge noninvariant) counterterm is given by

$$
\Gamma_{2}=\int \frac{d^{4} x \sqrt{g}}{(4 \pi)^{2}}\left(\frac{1}{3}\left(\nabla^{\mu} A^{\nu}\right)\left(\nabla_{\mu} A_{\nu}\right)+\frac{1}{6} R A^{2}\right)
$$


and the trace anomaly takes the form

$\left\langle T_{a}^{a}\right\rangle=-\frac{1}{48 \pi^{2}} F_{a b} F^{a b}$.

The counterterms $\Gamma_{1}$ and $\Gamma_{2}$ are consistent with each other, and merge into the unique counterterm (needed only at linear order in the metric)

$\Gamma_{3}=\int \frac{d^{4} x \sqrt{g}}{(4 \pi)^{2}}\left(\frac{2}{3} A^{4}+\frac{1}{3}\left(\nabla^{\mu} A^{v}\right)\left(\nabla_{\mu} A_{\nu}\right)+\frac{1}{6} R A^{2}\right)$

where, of course, $A^{2}=g^{\mu \nu} A_{\mu} A_{v}$ and $A^{4}=\left(A^{2}\right)^{2}$.

Thus, we have seen that the trace anomaly of a Weyl fermion does not contain any contribution from the topological density $F \tilde{F}$ (which on the other hand enters the chiral anomaly in (63), as well-known). It can be presented in a gauge invariant form by the variation of a local counterterm, and equals half the standard trace anomaly of a Dirac fermion. These are the main results of our paper.

\subsubsection{PV regularization with Dirac mass}

For using a Dirac mass we have to include a right handed free fermion in the PV lagrangian as well. The lagrangian is given in (24), and from Eq. (27) one finds the regulators

$$
\begin{aligned}
R_{\lambda} & =-\not \partial \not D(A) P_{L} \\
R_{\lambda_{c}} & =-\not \partial D(-A) P_{R} .
\end{aligned}
$$

Then, from the corresponding heat kernel coefficients $a_{2}$ we find the chiral anomaly

$\partial_{a}\left\langle J^{a}\right\rangle=\frac{1}{(4 \pi)^{2}}\left(\frac{1}{6} \varepsilon^{a b c d} F_{a b} F_{c d}-\frac{1}{3} \partial_{a}\left(A^{a} A^{2}\right)+\frac{1}{3} \square(\partial A)\right)$.

It contains noncovariant normal-parity terms, that are canceled by the variation of the local counterterm

$\Gamma_{4}=\int \frac{d^{4} x}{(4 \pi)^{2}}\left(\frac{1}{12} A^{4}-\frac{1}{6} A^{a} \square A_{a}\right)$

so that the anomaly takes the standard form

$\partial_{a}\left\langle J^{a}\right\rangle=\frac{1}{96 \pi^{2}} \varepsilon^{a b c d} F_{a b} F_{c d}$

as in the previous section.

Unfortunately, we cannot proceed to compute in a simple way the trace anomaly using this regularization, as the mass term breaks the Einstein and local Lorentz symmetries as well. The ensuing anomalies should then be computed and canceled by local counterterms, to find eventually the expected agreement of the remaining trace anomaly with the one found in the previous section.

\subsection{Chiral and trace anomalies of a Dirac fermion}

For completeness, we now consider the case of the massless Dirac spinor coupled to vector and axial gauge fields with lagrangian given in Eq. (31). The results are well-known, but we wish to present them for comparison and as a check on our method. The most natural regularization is obtained by employing a Dirac mass for the PV fields, but we employ also a Majorana mass. The latter allows to take a chiral limit in a simple way, which we use to rederive the previous results on the Weyl fermion.

\subsubsection{PV regularization with Dirac mass}

The relevant regulators are obtained from (40) and read

$$
\begin{aligned}
R_{\psi} & =-\not D(A, B)^{2} \\
R_{\psi_{c}} & =-\not D(-A, B)^{2} .
\end{aligned}
$$

The vector symmetry is guaranteed to remain anomaly free by the invariance of the mass term, while the chiral anomaly from (59) becomes

$$
\begin{aligned}
\partial_{a}\left\langle J_{5}^{a}\right\rangle= & \frac{1}{(4 \pi)^{2}}\left(\varepsilon^{a b c d} F_{a b}(A) F_{c d}(A)\right. \\
& +\frac{1}{3} \varepsilon^{a b c d} F_{a b}(B) F_{c d}(B) \\
& \left.-\frac{16}{3} \partial_{a}\left(B^{a} B^{2}\right)+\frac{4}{3} \square(\partial B)\right) .
\end{aligned}
$$

It contains normal-parity terms in the $B$ field. They are canceled by the variation of a local counterterm

$\Gamma_{5}=\int \frac{d^{4} x}{(4 \pi)^{2}}\left(\frac{4}{3} B^{4}-\frac{2}{3} B^{a} \square B_{a}\right)$

so that one ends up with

$$
\begin{aligned}
\partial_{a}\left\langle J^{a}\right\rangle= & 0 \\
\partial_{a}\left\langle J_{5}^{a}\right\rangle= & \frac{1}{(4 \pi)^{2}}\left(\varepsilon^{a b c d} F_{a b}(A) F_{c d}(A)\right. \\
& \left.+\frac{1}{3} \varepsilon^{a b c d} F_{a b}(B) F_{c d}(B)\right) .
\end{aligned}
$$

As for the trace anomaly, we find from (59)

$$
\begin{aligned}
\left\langle T_{a}^{a}\right\rangle= & -\frac{1}{(4 \pi)^{2}}\left(\frac{2}{3} F_{a b}(A) F^{a b}(A)\right. \\
& \left.+\frac{4}{3}\left(\partial_{a} B_{b}\right)\left(\partial^{a} B^{b}\right)-\frac{4}{3}(\partial B)^{2}-\frac{4}{3} \square B^{2}\right)
\end{aligned}
$$

with the counterterm

$$
\Gamma_{6}=\int \frac{d^{4} x \sqrt{g}}{(4 \pi)^{2}}\left(\frac{2}{3}\left(\nabla^{\mu} B^{v}\right)\left(\nabla_{\mu} B_{\nu}\right)+\frac{1}{3} R B^{2}\right)
$$


that brings it into the gauge invariant form

$$
\left\langle T^{a}{ }_{a}\right\rangle=-\frac{1}{24 \pi^{2}}\left(F_{a b}(A) F^{a b}(A)+F_{a b}(B) F^{a b}(B)\right) .
$$

All these counterterms merge naturally into the complete counterterm

$$
\Gamma_{7}=\int \frac{d^{4} x \sqrt{g}}{(4 \pi)^{2}}\left(\frac{4}{3} B^{4}+\frac{2}{3}\left(\nabla^{\mu} B^{\nu}\right)\left(\nabla_{\mu} B_{\nu}\right)+\frac{1}{3} R B^{2}\right) .
$$

\subsubsection{PV regularization with Majorana mass}

At last, we consider the regularization with a Majorana mass. As both vector and chiral symmetries are broken by the mass term, we expect anomalies in both $U(1)$ currents. From Eq. (43) we find the regulators

$$
\begin{aligned}
R_{\psi} & =-\not D(-A, B) \not D(A, B) \\
R_{\psi_{c}} & =-\not D(A, B) \not D(-A, B) .
\end{aligned}
$$

Then, we compute from (59)

$$
\begin{aligned}
\partial_{a}\left\langle J^{a}\right\rangle= & \frac{1}{(4 \pi)^{2}}\left(\frac{2}{3} \varepsilon^{a b c d} F_{a b}(A) F_{c d}(B)+\frac{4}{3} \square(\partial A)\right. \\
& \left.-\frac{16}{3} \partial_{a}\left[A^{a}\left(A^{2}+B^{2}\right)\right]-\frac{32}{3} \partial_{a}\left(B^{a} A_{b} B^{b}\right)\right)
\end{aligned}
$$

and

$$
\begin{aligned}
\partial_{a}\left\langle J_{5}^{a}\right\rangle= & \frac{1}{(4 \pi)^{2}}\left(\frac{1}{3} \varepsilon^{a b c d} F_{a b}(A) F_{c d}(A)\right. \\
& +\frac{1}{3} \varepsilon^{a b c d} F_{a b}(B) F_{c d}(B)+\frac{4}{3} \square(\partial B) \\
& \left.-\frac{16}{3} \partial_{a}\left[B^{a}\left(A^{2}+B^{2}\right)\right]-\frac{32}{3} \partial_{a}\left(A^{a} A_{b} B^{b}\right)\right) .
\end{aligned}
$$

The counterterm $\Gamma_{8}+\Gamma_{9}$

$$
\begin{aligned}
\Gamma_{8}= & \int \frac{d^{4} x}{(4 \pi)^{2}}\left(\frac{4}{3}\left(A^{2}+B^{2}\right)^{2}+\frac{16}{3}\left(A^{a} B_{a}\right)^{2}-\frac{2}{3} A^{a} \square A_{a}\right. \\
& \left.-\frac{2}{3} B^{a} \square B_{a}\right) \\
\Gamma_{9}= & \int \frac{d^{4} x}{(4 \pi)^{2}}\left(\frac{8}{3} \varepsilon^{a b c d} B_{a} A_{b}\left(\partial_{c} A_{d}\right)\right)
\end{aligned}
$$

allows to recover vector gauge invariance, and the anomalies take the form

$$
\partial_{a}\left\langle J^{a}\right\rangle=0
$$

$$
\begin{aligned}
\partial_{a}\left\langle J_{5}^{a}\right\rangle= & \frac{1}{(4 \pi)^{2}}\left(\varepsilon^{a b c d} F_{a b}(A) F_{c d}(A)\right. \\
& \left.+\frac{1}{3} \varepsilon^{a b c d} F_{a b}(B) F_{c d}(B)\right) .
\end{aligned}
$$

As for the trace anomaly, we find

$$
\begin{aligned}
\left\langle T_{a}^{a}\right\rangle= & -\frac{1}{(4 \pi)^{2}}\left(\frac{4}{3}\left(\partial_{a} A_{b}\right)\left(\partial^{a} A^{b}\right)-\frac{4}{3}(\partial A)^{2}-\frac{4}{3} \square A^{2}\right. \\
& \left.+\frac{4}{3}\left(\partial_{a} B_{b}\right)\left(\partial^{a} B^{b}\right)-\frac{4}{3}(\partial B)^{2}-\frac{4}{3} \square B^{2}\right)
\end{aligned}
$$

and using the counterterm

$$
\begin{aligned}
\Gamma_{10}=\int & \frac{d^{4} x \sqrt{g}}{(4 \pi)^{2}}\left(\frac{2}{3}\left(\nabla^{\mu} A^{v}\right)\left(\nabla_{\mu} A_{\nu}\right)+\frac{2}{3}\left(\nabla^{\mu} B^{v}\right)\left(\nabla_{\mu} B_{\nu}\right)\right. \\
& \left.+\frac{1}{3} R\left(A^{2}+B^{2}\right)\right)
\end{aligned}
$$

we get the final gauge invariant form

$$
\left\langle T_{a}^{a}\right\rangle=-\frac{1}{24 \pi^{2}}\left(F_{a b}(A) F^{a b}(A)+F_{a b}(B) F^{a b}(B)\right) .
$$

Also the counterterms employed in this section are consistent with each other, and combine into a unique final counterterm, which we report for completeness

$$
\begin{aligned}
\Gamma_{11}= & \int \frac{d^{4} x \sqrt{g}}{(4 \pi)^{2}}\left(\frac{2}{3}\left(\nabla^{\mu} A^{v}\right)\left(\nabla_{\mu} A_{v}\right)+\frac{2}{3}\left(\nabla^{\mu} B^{\nu}\right)\left(\nabla_{\mu} B_{v}\right)\right. \\
& +\frac{1}{3} R\left(A^{2}+B^{2}\right)+\frac{4}{3}\left(A^{2}+B^{2}\right)^{2}+\frac{16}{3}\left(A^{\mu} B_{\mu}\right)^{2} \\
& \left.+\frac{4}{3} \frac{\varepsilon^{\mu \nu \rho \sigma}}{\sqrt{g}} B_{\mu} A_{\nu} F_{\rho \sigma}(A)\right) .
\end{aligned}
$$

Evidently, the anomalies computed with the Majorana mass coincide with those obtained with the Dirac mass, after using local counterterms.

The results of this section can be projected consistently to recover the chiral and trace anomalies of a Weyl fermion. Indeed, one can consider the limit $A_{a}=B_{a} \rightarrow \frac{1}{2} A_{a}$. In this limit, a chiral projector $P_{L}=\frac{1+\gamma^{5}}{2}$ emerges inside the Dirac lagrangian (31) to reproduce the Weyl lagrangian (1). In addition, in the coupling to gravity, the right handed component of the Dirac field can be kept free, both in the kinetic and PV mass term, preserving at the same time covariance of the mass term of the left handed part of the PV Dirac fermion. Then, the right handed part decouples completely and can be ignored altogether. Thus, one may verify that the anomalies in Sect. 4.1.1 are reproduced by those computed here, including the counterterms, by setting $A_{a}=B_{a} \rightarrow \frac{1}{2} A_{a}$ (note that the current $J^{a}$ in Sect. 4.1.1 corresponds to half the sum of $J^{a}$ and $J_{5}^{a}$ of this section). 
As final remark, we have checked that terms proportional to $\delta \mathscr{O}$ in (54) never contribute to the anomalies computed thus far, as the extra terms vanish under the Dirac trace.

\section{Conclusions}

We have calculated the trace anomaly of a Weyl fermion coupled to an abelian gauge field. We have found that the anomaly does not contain any odd-parity contribution. In particular, we have shown that the Chern-Pontryagin term $F \tilde{F}$ is absent, notwithstanding the fact that it satisfies the consistency conditions for Weyl anomalies. Of course, the chiral anomaly implies that gauge invariance is broken. Nevertheless the trace anomaly can be cast in a gauge invariant form, equal to half the standard contribution of a nonchiral Dirac fermion.

While this result seems to have no direct implications for the analogous case in curved background, it strengthens the findings of ref. [6].

Recently, a generalized axial metric background has been developed in $[2,3]$ to motivate and explain the appearance of the Pontryagin term in the trace anomaly of a Weyl fermion, which however is in contradiction with the explicit calculation of [6]. Perhaps it would be useful to apply the methods used here in the axial metric background to clarify the situation in that context, and spot the source of disagreement.

Acknowledgements We thank Stefan Theisen for extensive discussions, suggestions, critical reading of the manuscript, and hospitality at AEI. In addition, FB would like to thank Loriano Bonora and Claudio Corianò for useful discussions, and $\mathrm{MB}$ acknowledges Lorenzo Casarin for interesting discussions and insights into anomalies and heat kernel.

Data Availability Statement This manuscript has associated data in a data repository. [Authors' comment: The datasets generated and analysed during the current study are available from the authors on reasonable request.]

Open Access This article is distributed under the terms of the Creative Commons Attribution 4.0 International License (http://creativecomm ons.org/licenses/by/4.0/), which permits unrestricted use, distribution, and reproduction in any medium, provided you give appropriate credit to the original author(s) and the source, provide a link to the Creative Commons license, and indicate if changes were made.

Funded by SCOAP ${ }^{3}$.

\section{Appendix A: Conventions}

We use a mostly plus Minkowski metric $\eta_{a b}$. The Dirac matrices $\gamma^{a}$ satisfy

$\left\{\gamma^{a}, \gamma^{b}\right\}=2 \eta^{a b}$

and the conjugate Dirac spinor $\bar{\psi}$ is defined using $\beta=i \gamma^{0}$ by

$\bar{\psi}=\psi^{\dagger} \beta$.
The hermitian chiral matrix $\gamma^{5}$ is given by

$\gamma^{5}=-i \gamma^{0} \gamma^{1} \gamma^{2} \gamma^{3}$

and used to define the chiral projectors

$P_{L}=\frac{\mathbb{1}+\gamma_{5}}{2}, \quad P_{R}=\frac{\mathbb{1}-\gamma_{5}}{2}$

that split a Dirac spinor $\psi$ into its left and right Weyl components

$\psi=\lambda+\rho, \quad \lambda=P_{L} \psi, \quad \rho=P_{R} \psi$.

The charge conjugation matrix $C$ satisfies

$C \gamma^{a} C^{-1}=-\gamma^{a T}$,

it is antisymmetric and used to define the charge conjugation of the spinor $\psi$ by

$\psi_{c}=C^{-1} \bar{\psi}^{T}$

where the roles of particle and antiparticle are interchanged. Note that a chiral spinor $\lambda$ has its charge conjugated field $\lambda_{c}$ of opposite chirality. A Majorana spinor $\mu$ is a spinor that equals its charged conjugated spinor

$\mu=\mu_{c}$.

This constraint is incompatible with the chiral constraint, and Majorana-Weyl spinors do not exist in 4 dimensions.

We find it convenient, as a check on our formulas, to use the chiral representation of the gamma matrices. In terms of $2 \times 2$ blocks they are given by

$\gamma^{0}=-i\left(\begin{array}{ll}0 & \mathbb{1} \\ \mathbb{1} & 0\end{array}\right), \quad \gamma^{i}=-i\left(\begin{array}{cc}0 & \sigma^{i} \\ -\sigma^{i} & 0\end{array}\right)$

where $\sigma^{i}$ are the Pauli matrices, so that

$\gamma^{5}=\left(\begin{array}{cc}\mathbb{1} & 0 \\ 0 & -\mathbb{1}\end{array}\right), \quad \beta=i \gamma^{0}=\left(\begin{array}{ll}0 & \mathbb{1} \\ \mathbb{1} & 0\end{array}\right)$.

The chiral representation makes evident that the Lorentz generators in the spinor space $M^{a b}=\frac{1}{4}\left[\gamma^{a}, \gamma^{b}\right]=\frac{1}{2} \gamma^{a b}$ take a block diagonal form

$M^{0 i}=\frac{1}{2}\left(\begin{array}{cc}\sigma^{i} & 0 \\ 0 & -\sigma^{i}\end{array}\right), \quad M^{i j}=\frac{i}{2} \varepsilon^{i j k}\left(\begin{array}{cc}\sigma^{k} & 0 \\ 0 & \sigma^{k}\end{array}\right)$

and do not mix the chiral components of a Dirac spinor (as $\gamma^{5}$ is also block diagonal). The usual two-dimensional Weyl spinors appear inside a four-dimensional Dirac spinor as follows

$\psi=\left(\begin{array}{l}l \\ r\end{array}\right), \quad \lambda=\left(\begin{array}{l}l \\ 0\end{array}\right), \quad \rho=\left(\begin{array}{l}0 \\ r\end{array}\right)$

where $l$ and $r$ indicate two-dimensional independent spinors of opposite chirality. In the chiral representation one may 
take the charge conjugation matrix $C$ to be given by

$C=\gamma^{2} \beta=-i\left(\begin{array}{cc}\sigma^{2} & 0 \\ 0 & -\sigma^{2}\end{array}\right)$

and satisfies

$C=-C^{T}=-C^{-1}=-C^{\dagger}=C^{*}$

(some of these relations are representation dependent). In the chiral representation the Majorana constraint (A.8) takes the form

$\mu=\mu_{c} \quad \rightarrow \quad\left(\begin{array}{l}l \\ r\end{array}\right)=\left(\begin{array}{c}i \sigma^{2} r^{*} \\ -i \sigma^{2} l^{*}\end{array}\right)$

which shows that the two-dimensional spinors $l$ and $r$ cannot be independent. The Majorana condition can be solved in terms of the single two-dimensional left-handed spinor $l$ by

$\mu=\left(\begin{array}{c}l \\ -i \sigma^{2} l^{*}\end{array}\right)$

which, evidently, contains the four-dimensional chiral spinors $\lambda$ and $\lambda_{c}$ defined by

$\lambda=\left(\begin{array}{l}l \\ 0\end{array}\right), \quad \lambda_{c}=\left(\begin{array}{c}0 \\ -i \sigma^{2} l^{*}\end{array}\right)$.

In a four-dimensional spinors notation one can write

$\mu=\lambda+\lambda_{c}$.

Alternatively, the Majorana condition can be solved in terms of the two-dimensional right-handed spinor $r$ by

$\mu=\left(\begin{array}{c}i \sigma^{2} r^{*} \\ r\end{array}\right)$

which contains the four-dimensional chiral spinors $\rho$ and $\rho_{c}$

$\rho=\left(\begin{array}{l}0 \\ r\end{array}\right), \quad \rho_{c}=\left(\begin{array}{c}i \sigma^{2} r^{*} \\ 0\end{array}\right)$

and $\mu=\rho+\rho_{c}$. This solution is of course the same as the previous one, as one identifies $\lambda=\rho_{c}$.

The explicit dictionary between Weyl and Majorana spinors shows clearly that the field theory of a Weyl spinor is equivalent to that of a Majorana spinor, as Lorentz symmetry fixes uniquely their actions, which are bound to be identical.

Finally, we normalize our $\varepsilon$ symbols by $\varepsilon_{0123}=-1$ and $\varepsilon^{0123}=1$, so that

$\frac{1}{4} \operatorname{tr}\left(\gamma^{5} \gamma^{a} \gamma^{b} \gamma^{c} \gamma^{d}\right)=i \varepsilon^{a b c d}$

\section{Appendix B: The heat kernel}

We consider an operator in flat $D$ dimensional spacetime of the form

$H=-\nabla^{2}+V$ with $V$ a matrix potential and $\nabla^{2}=\nabla^{a} \nabla_{a}$ constructed with a gauge covariant derivative $\nabla_{a}=\partial_{a}+W_{a}$ that satisfies

$\left[\nabla_{a}, \nabla_{b}\right]=\partial_{a} W_{b}-\partial_{b} W_{a}+\left[W_{a}, W_{b}\right]=\mathscr{F}_{a b}$.

The trace of the corresponding heat kernel is perturbatively given by

$$
\begin{aligned}
\operatorname{Tr}\left[J e^{-i s H}\right]= & \int d^{D} x \operatorname{tr}\left[J(x)\left\langle x\left|e^{-i s H}\right| x\right\rangle\right] \\
= & \int d^{D} x \frac{i}{(4 \pi i s)^{\frac{D}{2}}} \sum_{n=0}^{\infty} \operatorname{tr}\left[J(x) a_{n}(x, H)\right](i s)^{n} \\
= & i \int \frac{d^{D} x}{(4 \pi i s)^{\frac{D}{2}}} \operatorname{tr}\left[J ( x ) \left(a_{0}(x, H)\right.\right. \\
& +a_{1}(x, H) i s+a_{2}(x, H)(i s)^{2} \\
& \left.\left.+a_{3}(x, H)(i s)^{3}+\cdots\right)\right]
\end{aligned}
$$

where the symbol "tr" is the trace on the remaining discrete matrix indices, $J(x)$ is an arbitrary matrix function, and $a_{n}(x, H)$ are the so-called Seeley-DeWitt, or heat kernel, coefficients. They are matrix valued, and the first few ones are given by

$a_{0}(x, H)=\mathbb{1}$

$a_{1}(x, H)=-V$

$a_{2}(x, H)=\frac{1}{2} V^{2}-\frac{1}{6} \nabla^{2} V+\frac{1}{12} \mathscr{F}_{a b}^{2}$.

As $V$ is allowed to be a matrix, then $\nabla_{a} V=\partial_{a} V+\left[W_{a}, V\right]$, etc..

In the main text, the role of the hamiltonian $H$ is played by the various regulators $R$, and $i s \sim \frac{1}{M^{2}}$, see Eq. (55). In $D=4$ the $s$-independent term is precisely the one with $a_{2}(x, H)$, which is the coefficient producing the anomalies in 4 dimensions (we use a minkowskian set-up, but justify the heat kernel formulas by Wick rotating to euclidean time and back, when necessary).

More details on the heat kernel expansion are found in [20,21], where the coefficients appear with the additional coupling to a background metric. They have been recomputed with quantum mechanical path integrals in [24], a useful report is [25], while in [26] one may find the explicit expression for $a_{3}(x, H)$, originally calculated by Gilkey [27], which is relevant for calculations of anomalies in 6 dimensions.

\section{Appendix C: Sample calculations}

As an example of the calculations leading to the results of Sect. 4, we consider the case of the PV regularization with Majorana mass used for the Weyl model in Sect. 4.1.1. One regulator needed there is 
$R_{\lambda}=-\not D(-A) \not D(A) P_{L}$.

Neglecting the projector, which can be reinstated later, one cast it in the general form of Eq. (B.22). Expanding the covariant derivatives in the latter one finds

$$
\begin{aligned}
H= & -\nabla^{2}+V=-\partial^{a} \partial_{a}-2 W^{a} \partial_{a}-\left(\partial_{a} W^{a}\right) \\
& -W^{a} W_{a}+V .
\end{aligned}
$$

Similarly, by expanding $R_{\lambda}$ one finds (up to the projector)

$$
\begin{aligned}
R_{\lambda} & =-\not D(-A) \not D(A) \\
& =-D^{a}(-A) D_{a}(A)-2 i \gamma^{a b} A_{a} \partial_{b}+\frac{i}{2} F_{a b} \gamma^{a b} \\
& =-\partial^{a} \partial_{a}+2 i \gamma^{a b} A_{b} \partial_{a}+i\left(\partial^{a} A_{a}\right)-A^{a} A_{a}+\frac{i}{2} F_{a b} \gamma^{a b}
\end{aligned}
$$

where $\gamma^{a b}=\frac{1}{2}\left[\gamma^{a}, \gamma^{b}\right]$. Comparing (C.27) and (C.28) one fixes

$$
\begin{aligned}
W^{a} & =-i \gamma^{a b} A_{b} \\
V & =2 A^{a} A_{a}+i\left(\partial^{a} A_{a}\right) .
\end{aligned}
$$

At this stage one proceeds to evaluate the field strength $\mathscr{F}_{a b}$ in Eq. (B.23) associated to this particular $W^{a}$, and use it to evaluate the coefficient $a_{2}\left(R_{\lambda}\right)$ from $a_{2}(H)$ of Eq. (B.25) (remembering to reinsert the projector). In particular, evaluating the trace one finds

$$
\begin{aligned}
\operatorname{tr}[ & \left.P_{L} a_{2}\left(R_{\lambda}\right)\right] \\
= & \frac{2}{3}\left(\partial_{a} A_{b}\right)\left(\partial^{a} A^{b}\right)-\frac{2}{3}(\partial A)^{2}-\frac{2}{3} \square A^{2} \\
& +i\left(\frac{4}{3} \partial_{a}\left(A^{a} A^{2}\right)-\frac{1}{3} \square(\partial A)-\frac{1}{12} \varepsilon^{a b c d} F_{a b} F_{c d}\right) .
\end{aligned}
$$

Note that this particular coefficient contains an odd-parity term proportional to the topological density $F \tilde{F}$. Similarly, one computes the coefficients related the other regulators, and proceeds to evaluate (56) and (59).

We have checked our trace calculations on the gamma matrices also by computer, employing a notebook developed in [28] using the xAct and xTensor packages [29,30].

\section{References}

1. L. Bonora, S. Giaccari, B. Lima de Souza, Trace anomalies in chiral theories revisited. JHEP 1407, 117 (2014). arXiv:1403.2606 [hep-th]

2. L. Bonora, M. Cvitan, P. Dominis Prester, A. Duarte Pereira, S. Giaccari, T. Štemberga, Axial gravity, massless fermions and trace anomalies. Eur. Phys. J. C 77(8), 511 (2017). arXiv:1703.10473 [hep-th]

3. L. Bonora, M. Cvitan, P. Dominis Prester, S. Giaccari, M. Paulišić, T. Štemberga, Axial gravity: a non-perturbative approach to split anomalies. Eur. Phys. J. C 78(8), 652 (2018). arXiv:1807.01249 [hep-th]
4. Y. Nakayama, CP-violating CFT and trace anomaly. Nucl. Phys. B 859, 288 (2012). arXiv:1201.3428 [hep-th]

5. Y. Nakayama, Realization of impossible anomalies. Phys. Rev. D 98(8), 085002 (2018). arXiv:1804.02940 [hep-th]

6. F. Bastianelli, R. Martelli, On the trace anomaly of a Weyl fermion. JHEP 1611, 178 (2016). arXiv:1610.02304 [hep-th]

7. L. Alvarez-Gaume, E. Witten, Gravitational anomalies. Nucl. Phys. B 234, 269 (1984)

8. H. Godazgar, H. Nicolai, A rederivation of the conformal anomaly for spin- $\frac{1}{2}$. Class. Quant. Grav 35(10), 105013 (2018). arXiv:1801.01728 [hep-th]

9. Y.S. Stanev, Correlation functions of conserved currents in four dimensional conformal field theory. Nucl. Phys. B 865, 200 (2012). arXiv: 1206.5639 [hep-th]

10. A. Zhiboedov, "A note on three-point functions of conserved currents," arXiv:1206.6370 [hep-th]

11. L. Bonora, A. Duarte Pereira, B. de Lima Souza, Regularization of energy-momentum tensor correlators and parity-odd terms. JHEP 1506, 024 (2015). arXiv:1503.03326 [hep-th]

12. W.A. Bardeen, Anomalous Ward identities in spinor field theories. Phys. Rev. 184, 1848 (1969)

13. L. Alvarez-Gaume, P. H. Ginsparg, "The structure of gauge and gravitational anomalies," Annals Phys. 161, 423 (1985) (Erratum: [Annals Phys. 171 (1986) 233])

14. W.A. Bardeen, B. Zumino, Consistent and covariant anomalies in gauge and gravitational theories. Nucl. Phys. B 244, 421 (1984)

15. W. Pauli, F. Villars, On the invariant regularization in relativistic quantum theory. Rev. Mod. Phys. 21, 434 (1949)

16. A. Diaz, W. Troost, P. van Nieuwenhuizen, A. Van Proeyen, Understanding Fujikawa regulators from Pauli-Villars regularization of ghost loops. Int. J. Mod. Phys. A 4, 3959 (1989)

17. M. Hatsuda, P. van Nieuwenhuizen, W. Troost, A. Van Proeyen, The regularized phase space path integral measure for a scalar field coupled to gravity. Nucl. Phys. B 335, 166 (1990)

18. K. Fujikawa, Path integral measure for gauge invariant fermion theories. Phys. Rev. Lett. 42, 1195 (1979)

19. K. Fujikawa, Comment on chiral and conformal anomalies. Phys. Rev. Lett. 44, 1733 (1980)

20. B. S. DeWitt, "Dynamical theory of groups and fields," Conf. Proc. C 630701 (1964) 585 [Les Houches Lect. Notes 13 (1964) 585]

21. B. S. DeWitt, The spacetime approach to quantum field theory. In: Relativity, groups and topology II, proceedings of the Les Houches Summer School 1983, edited by B.S. De Witt and R. Stora, North Holland, Amsterdam (1984)

22. J. Wess, B. Zumino, Consequences of anomalous Ward identities. Phys. Lett. 37B, 95 (1971)

23. L. Bonora, P. Cotta-Ramusino, C. Reina, Conformal anomaly and cohomology. Phys. Lett. 126B, 305 (1983)

24. F. Bastianelli, P. van Nieuwenhuizen, Trace anomalies from quantum mechanics. Nucl. Phys. B 389, 53 (1993). arXiv:hep-th/9208059

25. D.V. Vassilevich, Heat kernel expansion: User's manual. Phys. Rept. 388, 279 (2003). arXiv:hep-th/0306138

26. F. Bastianelli, S. Frolov, A.A. Tseytlin, Conformal anomaly of $(2,0)$ tensor multiplet in six-dimensions and AdS / CFT correspondence. JHEP 0002, 013 (2000). arXiv:hep-th/0001041

27. P .B. Gilkey, The spectral geometry of a Riemannian manifold. J. Diff. Geom 10(4), 601 (1975)

28. C. Pitrou, Clifford_Algebra_Traces.nb (2018). https://github.com/ xAct-contrib/examples/blob/master/README.md

29. J. M. Martín-García et al., "xAct: Efficient tensor computer algebra for the Wolfram Language," (2002-2018). http://xact.es/

30. J. M. Martín-García, xTensor: Fast abstract tensor computer algebra (2002-2018). http://xact.es/xTensor/ 